\title{
Quelques exemples d'application de la méthode de l'hydrogramme unitaire à des bassins versants expérimentaux d'Outre-mer
}

\section{Examples of applications of the unit hydrograph method to experimental catchment areas overseas}

\author{
PAR J. RODIER
}

INGENIEUR EN GHEF A L'ÉLECTRTGITÉ DE FRANCE

CHEF DU SERVICE HYDROLOGIQUE DE L'OFFICE DE LA RECHERCHE SCIENTIFIQUE ET TECHNIQUE OUTRE-MEI

\begin{abstract}
L'application des méthodes d'hydrologie analytique présente un certain nombre de difficnltés pratiques dues surtout à l'hétérogénéité des précipitations, all caractère complexe des diagrammes d'intensité des précipitations et all manque d'homogénéité des terrains. Les cludes des has. sins expérimentaux d'Outre-Mer montrent conment on peut résondre ces difficultés.

Il s'agit d'obtenir l'hydrogramme de crue pour une averse définie par ses diverses caractéristiques.

La méthode des hydrogrammes unitaires conduit assez facilement a l'hydrogramme type du bassin.

Par contre, la recherche de la relation entre hanteur de précipitations et volume de la crue correspondante, pour les divers états de saturation et pour les divers typ's de sols, est beaucoup plus délicate; on parvient a approcher cette relation par divers procédes.

Quelques exemples de calculs de crues décennales sont donnés en conclusion.
\end{abstract}

\begin{abstract}
A certain number of practical difficulties arise in the application of analytical hydrolog!y methods, resulting especially from the heterogeneous nuture of the local precipitations, the complex precipitation intensity diagrans involved, and the lack of homogeneity of the locat ground. Studies of experimental catchment areas overseas show how these difficulties can be overcome.

The aim is to determine the flood hydrograph for a shower defined $b y$ its various characteristics.

The unit hydrograph method provides a fairly convenient means of determining the typical hydrograph for the catchment area.

A much more delicate problem. on the other hand, is the determination of the relationship between rainfall and the corresponding flood volume, for various states of saturation and soil types. Various procedures are used in a surcessful approach to this relationship.

The paper ends with a few examples of tenyearly flood calculations.
\end{abstract}

La théorie des hydrogrammes unitaires s'intègre dans la méthode analytique d'étude des phénomènes de l'écoulement.

Pour une crue donnée sur un petit bassin, trois points sont à examiner :

- l'averse qui a donné naissance à la crue,

- le volume écoulé qui en est résulté,

- le débit maximal correspondant à ce volume.

L'étude des averses est relativement facile si la masse des observations pluviométriques est suffisante.
L'application des diverses recettes des hydrogrammes unitaires permet de résoudre la troisième question de façon satisfaisante dans de très nombreux cas.

La seconde est redoutable, mais les études d'hydrologie analytique qui se sont développées parallèlement à la théorie des hydrogrammes unitaires et, notamment, les observations systématiques des bassins expérimentaux, permettent d'aboutir souvent à des résultats acceptables.

Les méthodes d'hydrologie analytique comportent deux étapes: 
Dans un premier stade, on analyse averses et crues le plus souvent sur un bassin expérimental puis, dans une deuxième étape, grâce aux caractéristiques que cet examen a mises en évidence, notamment le diagramme de distribution et les variations de la capacité d'absorption avec l'importance de l'averse, on reconstitue l'hydrogramme de crue pour une averse donnée.

Si les études hydrologiques ont été suffisamment poussées, on peut réduire de beaucoup la première partie de cette étude et même se contenter d'un rapide examen du terrain. Dans certains cas, il suffit d'utiliser des courbes et des abaques. Mais nous n'en sommes pas là!

On roit que cette méthode peut s'appliquer à l'estimation de crues exceptionnelles, mais elle ne se limite pas là. Nous ne considérerons, cependant, dans ce qui suit, que cette étude particulière.

\section{I. - ANALYSE DES DONNEES D'OBSERVATIONS}

Généralement, elles ont été recueillies sur des bassins expérimentaux dont nous rappelons, cidessous, l'équipement : $1^{\circ}$ Un ensemble de pluviomètres (dix à vingt) et de pluviographes (au moins deux) destinés, pour chaque averse, à la détermination des ré-

\section{DANS L'ESPACE}
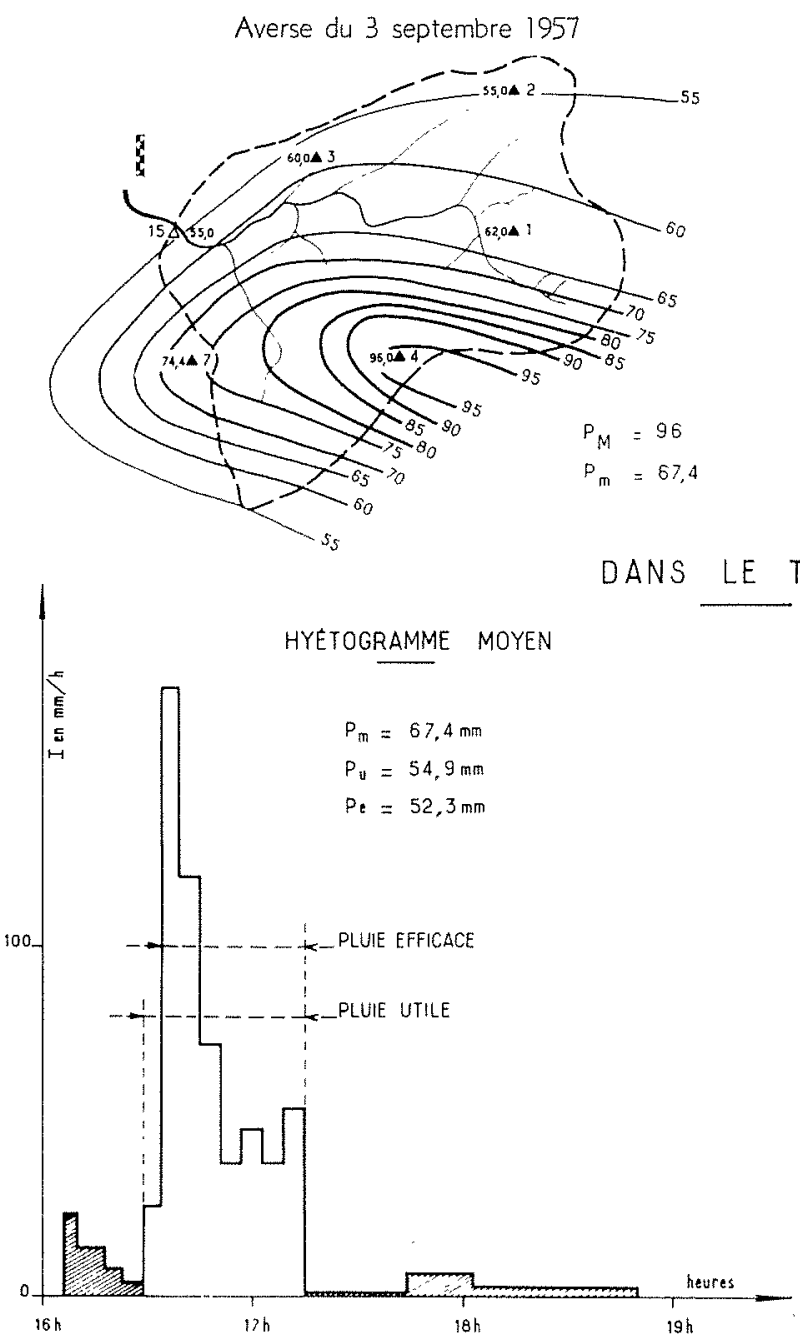
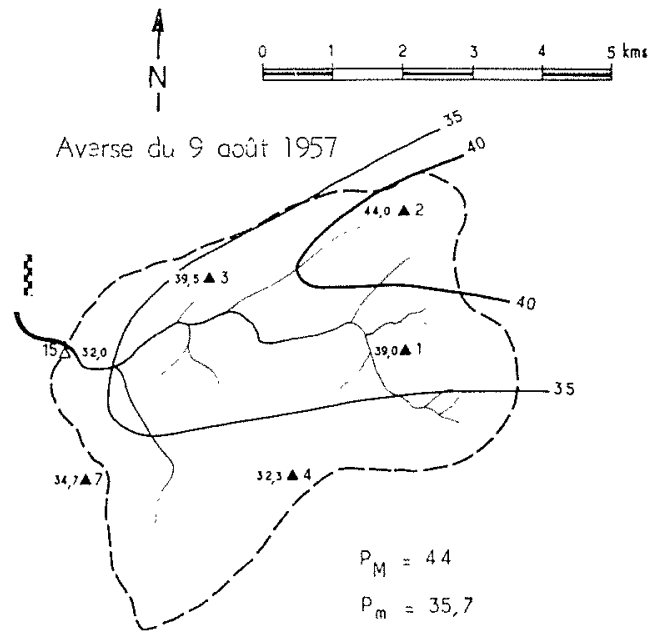

AVERSE $N^{\circ} 25$

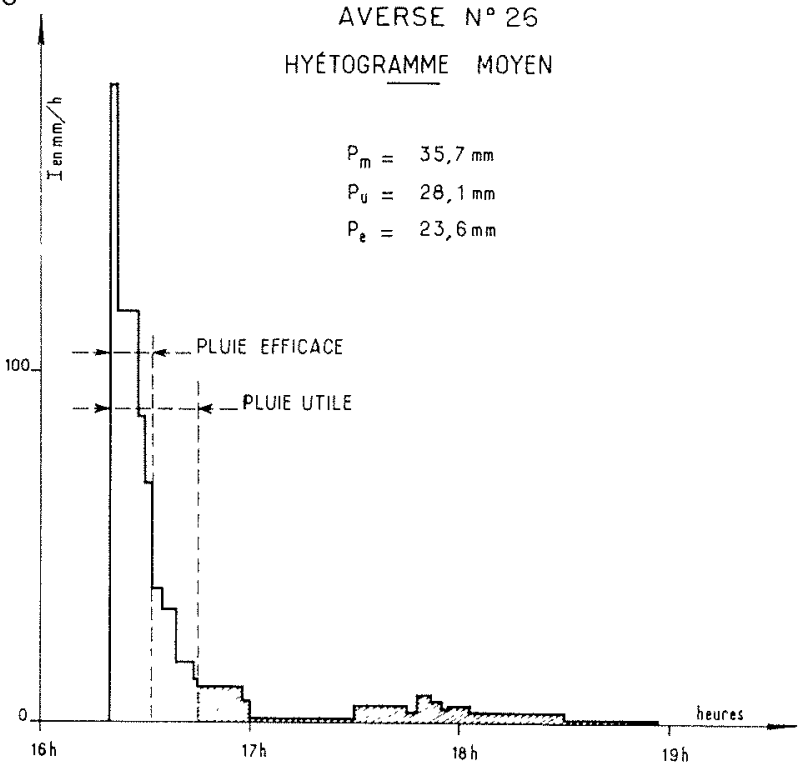

FIG. 1

Répartition de la pluie. Bassin versant de Koumbaka. 
partitions de la pluie dans le temps et dans l'espace;

$2^{\circ}$ Une ou plusieurs stations de jaugeages permettant d'établir, pour chaque crue, le diagramme des débits ou hydrogrammes.

Des mesures d'infiltrations et bien d'autres observations sont effectuées sur un bassin expérimental; leur énumération nous entraînerait trop loin.

L'analyse des données d'observations porte sur les averses et les crues :

\section{a) Etude des averses :}

\section{Elle doit :}

- caractériser chaque précipitation par un petit nombre de grandeurs en fonction desquelles il sera possible d'étudier les différents facteurs de la crue qui lui correspond;

- compléter les données des postes pluviométriques des Services météorologiques en vue de l'étude statistique complète des averses.

Chaque averse est caractérisée par la répartilion de la hauteur pluviométrique dans l'espace, la répartition des intensités dans le temps, et l'état de saturation du sol.

La répartition dans l'espace est donnée par le réseau d'isoyètes : deux exemples sont présentés par la figure 1 relative au bassin de Koumbaka au Mali. Ce réseau est traduit sur les tableaux comparatifs par trois chiffres qu'on trouvera sur le tableau 10 : le maximum ponctuel $\mathrm{P}_{M}$, la précipitation moyenne sur le bassin $P$ et une indication de la dispersion qui peut être indiquée très simplement par la valeur minimale $\mathrm{P}_{m}$. On retrouve l'averse 26 qui figurait sur le graphique 1.

Nous rappelons que la méthode des hydrogrammes unitaires n'est applicable que si la répartition n'est pas trop hétérogène. Si cette condition est remplie, l'averse réelle sera remplacée par une averse homogène de hauteur égale à la moyenne P. Mais l'examen de la dispersion et de la position de l'épicentre permet d'expliquer bien des anomalies.

Le graphique 1 montre deux lypes de répartilion des intensités dans le temps. On vérifie d'abord si les diagrammes des deux pluviographes sont assez voisins, sinon la méthode des hydrogrammes unitaires ne s'applique pas. Ce point étant vérifié, on compose ces diagrammes pour obtenir un diagramme moyen applicable a tout le bassin. Cette composition, dans les cas les plus simples, peut s'effectuer par la moyenne des intensités. Dans certains cas, il peut y avoir intérêt à employer la méthode Rochette expliquée par la figure 2.

Les intensités de chaque pluviographe sont
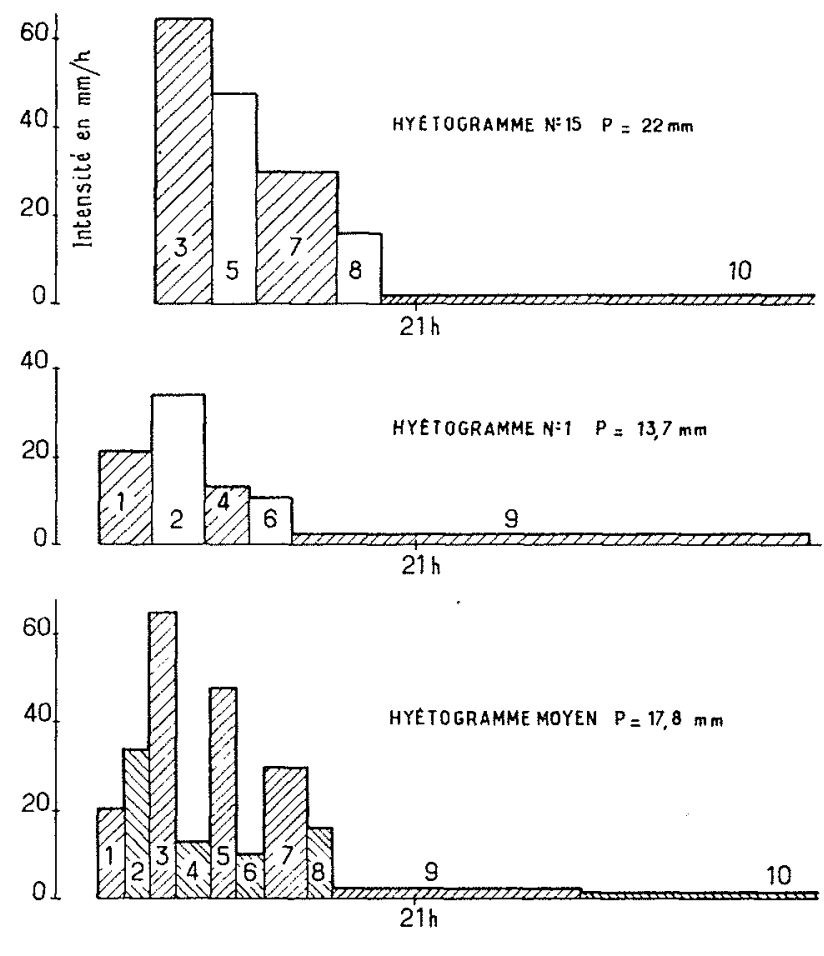

Frg. 2

Composition du hyétogramme moyen d'une averse. Koumbaka, le 31-7-56.

conservées, les durées correspondantes sont réduites en fonction du poids affecté à chaque pluviomètre; on évite ainsi d'éliminer des pointes locales d'intensité qui donnent lieu à ruissellement. Enfin, dans les cas les plus complexes, on peut employer la méthode de Horton.

La figure 1 montre que certaines parties du hyétogramme ne donnent pas lieu manifestement au ruissellement, seule la partie centrale ou corps de l'averse intervient. On est ainsi amené à définir une pluie utile correspondant à ce corps ou une pluie efficace qui correspond cxactement à la durée du ruissellement.

Un hyétogramme peut donc être caractérisé par une série de chiffres: hauteur totale de prócipitations, hauteur utile on hateur efficace, inlensité maximale, intensité utile, durée utile, etc.

Une partie de ces caracléristiques, souvent la hauteur utile et l'inlensité maximale, sonl portées pour les hyélogrammes moyens de chaque averse sur des tableaux tels que le lableau 11 .

Enfin, pour caractériser l'état de saturation du sol, on précise, en heures ou en jours, l'intervalle séparant l'averse étudiée de la première précipitation antérieure dépassant 2 ou $5 \mathrm{~mm}$. Il est parfois intéressant d'indiquer par un signe spécial si cette averse antérieure a ćté faible, médiocre, forte ou très forte. On peut remplacer ces deux données par un indice de saturation qui en est une fonction simple.

Pour les bassins perméables, le débit de basc $Q_{0}$ 
ou début de la crue peut fournir une bonne indication sur l'état de saturation.

\section{b) Etude des crues :}

L'étude des crues a pour objet la détermination de la forme type de l'hydrogramme ou diagramme de distribution et de la valeur de la capacité d'absorption ou du coefficient de ruissellement en fonction des diverses caractéristiques de l'averse telles qu'elles viennent d'être définies.

\section{$1^{\circ}$ Détermination du diagramme de DISTRIBUTION :}

Des relevés des limnigraphes et de la courbe d'étalonnage, on déduit les hydrogrammes bruts.

Il s'agit, sur ceux-ci, de séparer le ruissellement des autres formes de l'écoulement. Précisons à ce sujet que, si le ruissellement est classique en terrain imperméable avec la formation d'un film et, parfois, d'une nappe d'eau super-

ISOHYÈTES pour l'averse du 20 juillet 1956

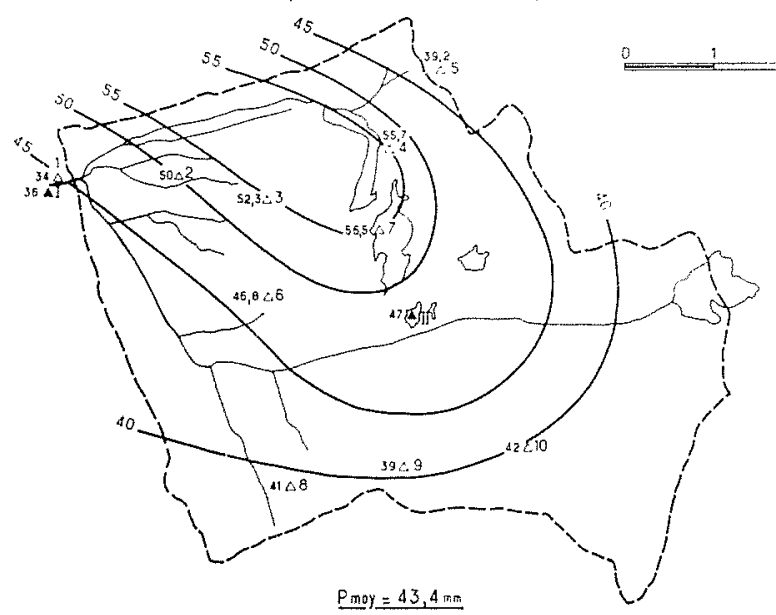

$P_{\text {moy }}=43,4 \pi \pi$

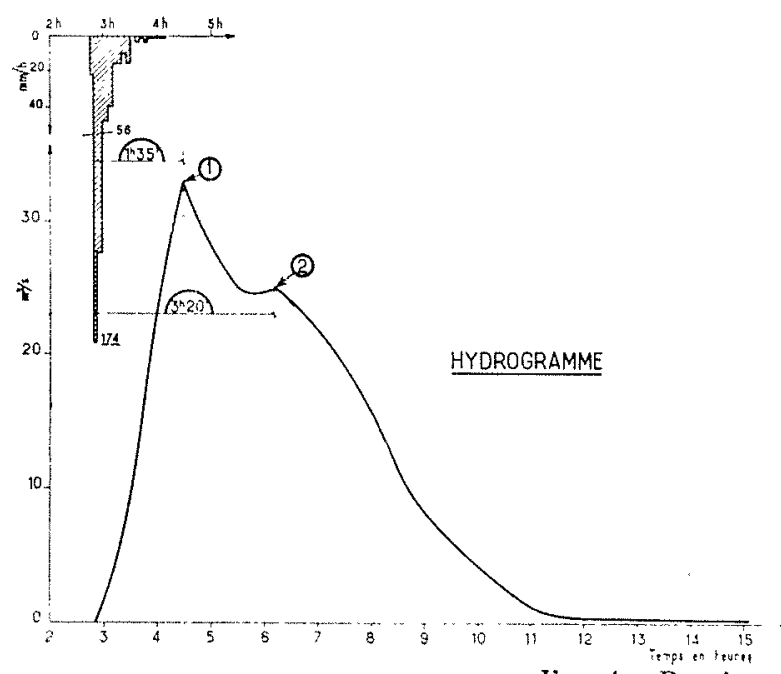

Jis, 4 Bassin versant de Tin Adjar.
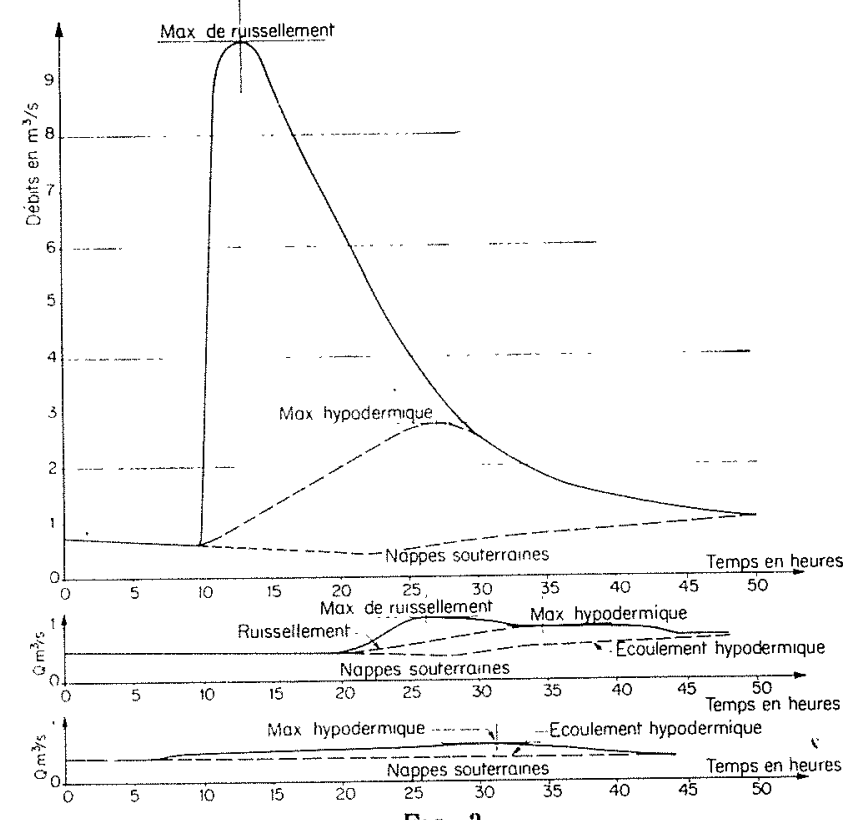

Fig. 3

Différentes formes d'écoulement sur un bassin versant.

ISOHYÈTES pour l'overse du 12 août 1956
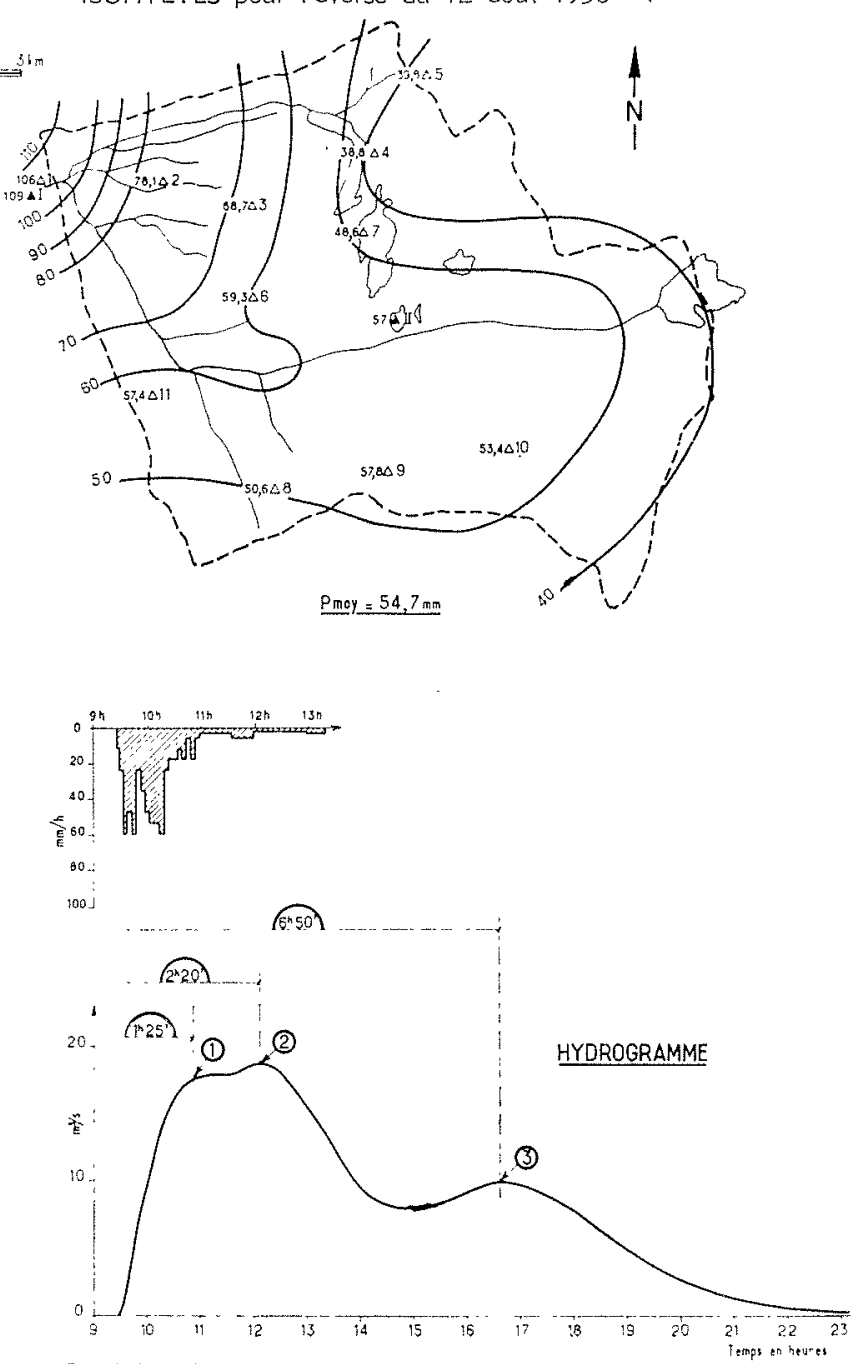
ficielle continue, dans le cas de terrains perméables ou de régions forestières, ce que l'on serait tenté d'appeler ruissellement est, en fait, presque de l'éconlement hypodermique.

La séparation entre ruissellement et écoulement hypodermique est parfois malaisée, comme on peut le voir sur la figure 3 , relative à un bassin de savane boisée de Côte-d'Tvoire, sur lequel l'origine des temps correspond au centre de gravité de l'averse. On voit que l'examen simultané d'hydrogrammes de diverses importances et, surlout, le délai entre précipitation et maximum, permettent de séparer les formes de l'écoulement; l'indication classique du changement de courbure lans la courbe de récession n'est pas suffisante dans de nombreux cas. Une indication supplémentaire est donnée par le fait que les maximums hypodermiques s'écartent peu les uns des autres et que le débit maximal croît avec le volume total de la crue. Il est important de tracer tous les hydrogrammes à la même échelle.

Pour obtenir, à partir de ces hydrogrammes de ruissellement, l'hydrogramme type ou diagramme de distribution, il convient d'éliminer les averses non unitaires. II faut que la durée de la pluie utile soit inférieure à une limite, fonction du temps de concentration ou du temps de montée. Mais les valeurs expérimentales de celui-ci présentent une notable dispersion due, soit à des averses très localisées, soit à des hyétogrammes complexes. Par exemple, sur un bassin de $4 \mathrm{~km}^{2}$ du Nord-Cameroun, les 38 valeurs du temps de montée sont comprises entre $5 \mathrm{mn}$ et $30 \mathrm{mn}$. Mais si l'on ne considère que les averses de faible durée, on trouve des valeurs comprises entre 5 et $9 \mathrm{mn}$. On retiendra un temps de montée compris entre 7 et $8 \mathrm{mn}$. Théoriquement, devrait être considérée comme unitaire toute averse de durée utile inférieure à $4 \mathrm{mn}$; en fait, on peut se montrer un peu plus large.

Enfin, il faut éliminer du diagramme de ruissellement toute irrégularité due aux pointes d'aver'se secondaires. Mais il convient d'être très prudent, car certains hydrogrammes présentent systémaliquement des bosses dues à l'arrivée d'affluents secondaires près de la station. Le cas extrême est représenté par le graphique 4 relatif au bassin de 'Tin Adjar dans la boucle du Niger, pour lequel le diagramme type sur la partie droite de la figure comporte quatre pointes, ce qui exige des méthodes de dépouillement spéciales qui nous entraîneraient trop loin.

Le volume de ruissellement est alors déterminé sur chaque diagramme de ruissellement et on reproduit alors ces diagrammes pour un même volume unitaire de $10000 \mathrm{~m}^{3}, 50000$ ou $100000 \mathrm{~m}^{3}$ en divisant les ordonnées des diagrammes de ruissellement dans le rapport $V_{\mathrm{R}} / 10000$, par exemple.
On obtient ainsi une série de diagrammes de distribution tels que le diagramme 5 , qui sont lous très voisins les uns des autres en général, et on adopte le diagramme moyen. On peut schématiser le diagramme par un escalier de 5 ou 10 marches, mais, en pratique, il est possible de procéder beaucoup plus simplement sans avoir à dessiner un seul hydrogramme de ruissellement.

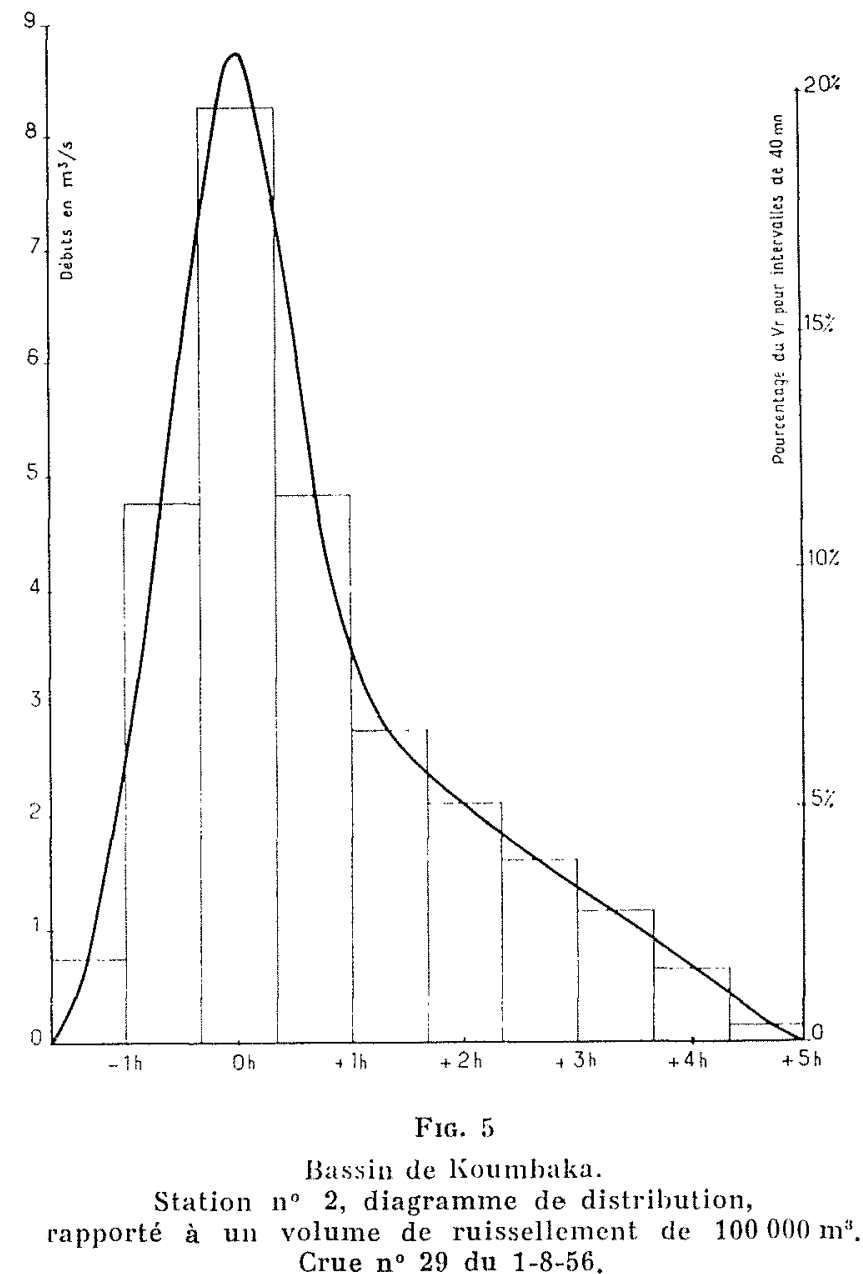

On onère ainsi sur l'hydrogramme brut: on sépare le ruissellement, on détermine le maximum de ruissellement et on reporte sur un tableau les débits à des intervalles de temps donnés, avant et après le maximum, en réduisant ces débits de façon à ce qu'ils correspondent à un volume de ruissellement donné. On obtient ainsi un tableau tel que le tableau $\mathrm{n}^{\circ} 6$.

La dispersion sur les valeurs du temps de ruissellement est grande, ce qui résulte des difficultés de séparation entre écoulement hypodermique et ruissellement est sans importance.

A noter qu'en forêt, on peut obtenir deux tyyes de diagrammes de distribution correspondant à 
Tableau 6

\begin{tabular}{|c|c|c|c|c|c|c|c|c|c|c|}
\hline $\mathrm{N}^{\circ}$ des crues & $-1 \mathrm{~h}$ & $-45 \mathrm{mn}$ & $-30 \mathrm{mn}$ & $-15 \mathrm{mn}$ & $\begin{array}{c}\mathrm{Q} \\
Q_{\max }\end{array}$ & $+15 \mathrm{mn}$ & $+30 \mathrm{mn}$ & $+1 \mathrm{~h}$ & $+2 \mathrm{~h}$ & $+3 \mathrm{~h}$ \\
\hline 26 & 1,6 & 4,8 & 6,4 & 7,3 & 8,2 & 7,9 & 7,7 & 7 & 3,8 & 2,2 \\
\hline 29 & 2,2 & 4 & 6,3 & 7,8 & 8,7 & 7,4 & 5,6 & 3,3 & 2,1 & 1,5 \\
\hline 32 & 1,4 & 5,9 & 7,1 & 7,4 & 7,7 & 7,3 & 7,1 & 5,9 & 3,6 & 2,4 \\
\hline $\begin{array}{c}\text { Hydrogramme } \\
\text { moyen }\end{array}$ & 1,1 & 4,4 & 6,7 & 8,2 & 9 & 8,4 & 7,5 & 5,7 & 3,1 & 2,1 \\
\hline
\end{tabular}

Bassin de Koumbaka - Station $\mathrm{n}^{\circ} 2$

Tableau des hydrogrammes unitaires de ruissellement

Débit pour $\mathrm{V} r=1 / 100000$

deux modes différents d'écoulement; ce sont les diagrammes les plus aigus qu'il faut prendre. A ce stade des études, nous ne conseillons pas de remplacer les hydrogrammes par des triangles comme cela a été suggéré : la mauvaise détermination du temps de ruissellement conduirait à de graves erreurs.

\section{$2^{\circ}$ DÉTERMination DU VOLUME DE RUISSELLEMEN'T POUR UNE AVERSE DONNÉE :}

C'est la partie la plus délicate et la moins précise de l'étude. Il s'agit de calculer la part du ruissellement en déduisant les pertes par interception, par mise en réserve à la surface du sol et par infiltration. Les pertes par évaporation immédiate sont faibles.

On peut effectuer cette opération :

- soit en chiffrant l'ensemble des pertes par unité de temps : on obtient ainsi la capacité d'absorption, qu'il est plus honnête d'appeler « capacité d'absorption apparente », car la capacité d'absorption instantanée est pratiquement inaccessible;

- soit en déterminant le rapport du volume de ruissellement au volume de l'averse, c'està-dire le coefficient de ruissellement $K_{12}$.

On simplifie un peu le problème en remplaçant l'averse réelle par la pluie utile $P_{u}$ et le coefficient de ruissellement utile $\mathrm{K}_{r u}=\mathrm{V}_{\mathrm{R}} / \mathrm{V}_{\mathrm{P} u}$, ou par la pluie efficace et $\mathrm{K}_{\text {re }}$.

$\mathrm{Si}$ le bassin est peu perméable et la couverture végétale pas très dense, on détermine la capacité d'absorption en portant au sommet du hyétogramme moyen la hauteur de précipitation égale à la lame d'eau de ruissellement, comme sur l'exemple de la figure 7 où l'averse 13 a fourni un volume de ruissellement de $25000 \mathrm{~m}^{3}$ correspondant à $1 \mathrm{~mm}$. La capacité d'absorption est l'ordonnée de la ligne horizontale limitant vers le bas cette hauteur nette, soit, ici, $55 \mathrm{~mm} / \mathrm{h}$.

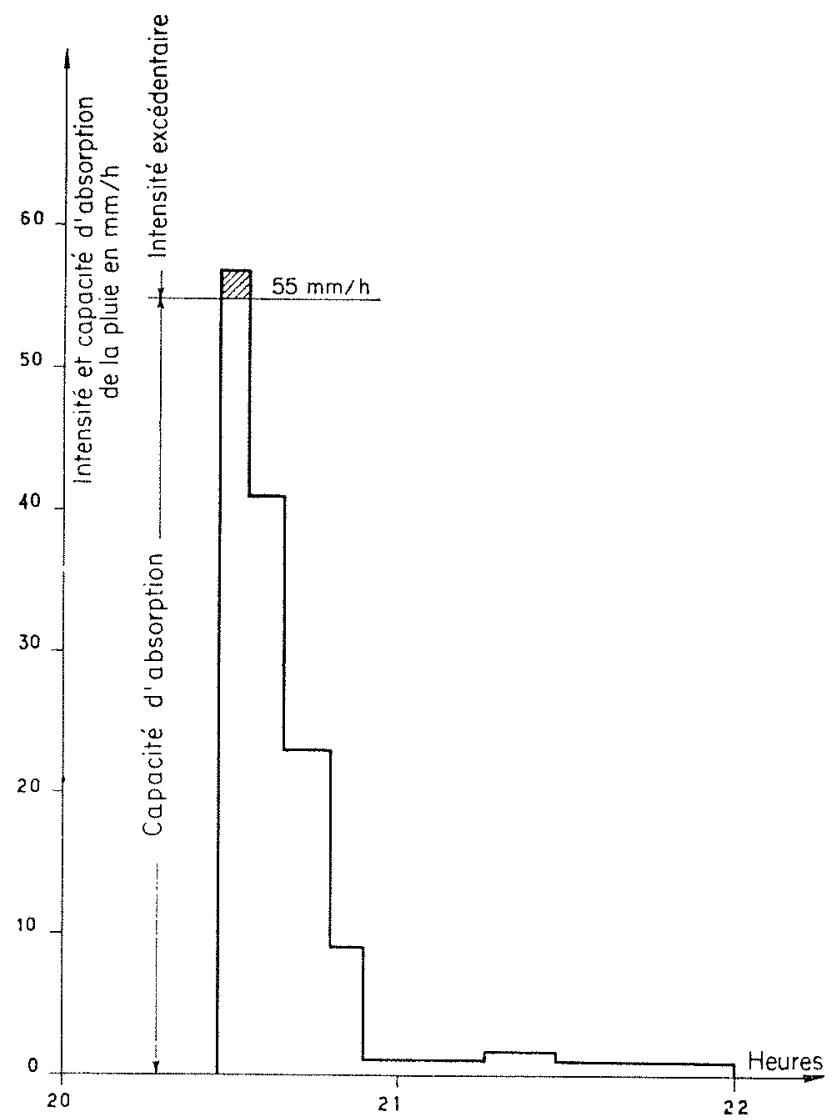

FIG. 7

Bassin versant expérimental de Koumbaka It. Détermination de la capacité d'absorption. Averse $11^{*} 13$. 


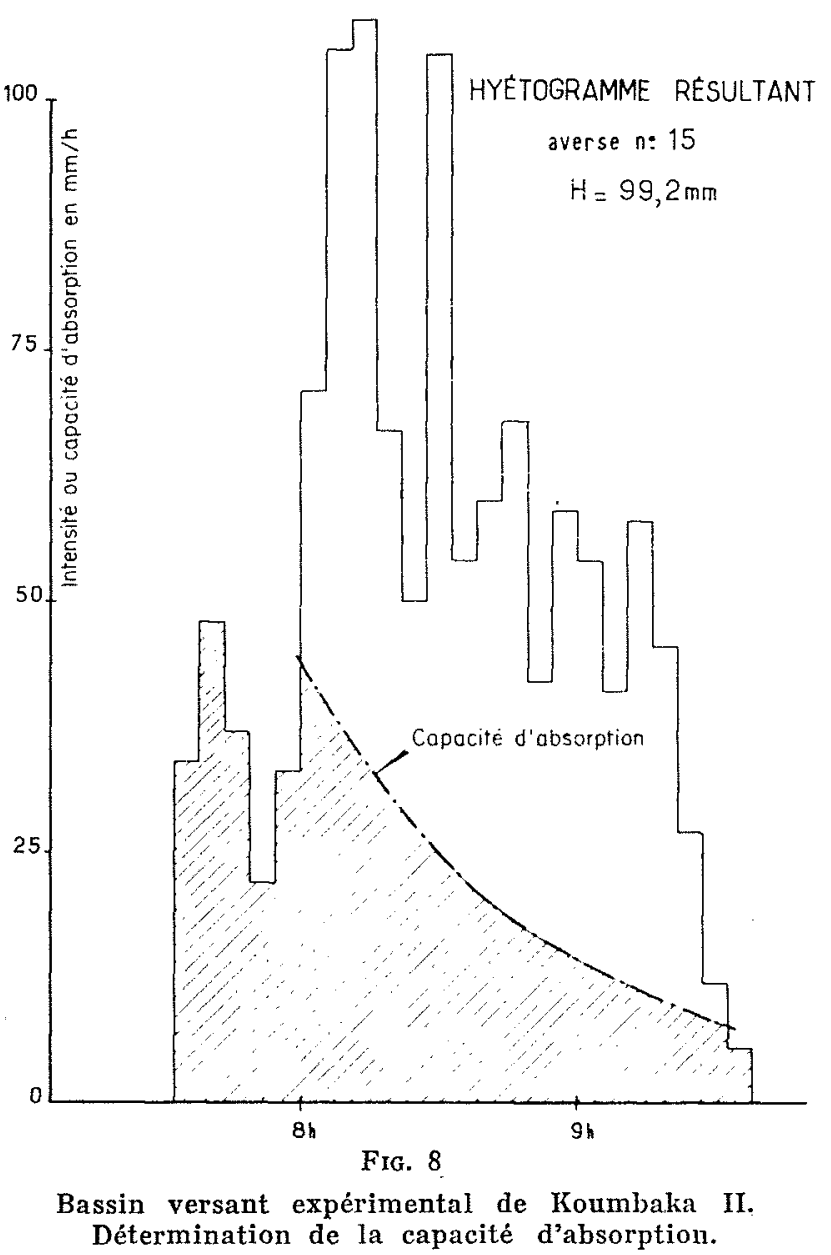

Si l'averse est d'assez longue durée, la capaeité d'absorption décroît avec le temps et la ligne horizontale doit être remplacée par une courbe décroissante comme sur la figure 8. Les deux extrémités de la courbe sont difficiles à déterminer; on utilise pour le début des averses très brèves et, pour la fin, les dernières pointes d'averses multiples telles que celles de la figure 9.

Pour les bassins forestiers, la couverture végétale régularise en fait le hyétogramme et on a intérêt à dessiner ce dernier à partir d'intervalles de temps de 15 à 20 minutes et non de 2 à 5 minutes comme en terrain dégagé, sinon on trouverait des capacités d'absorption beaucoup trop fortes.

Pour les terrains perméables, il y a intérêt à utiliser une méthode mise au point par A. Bouchardeau : on détermine la hauteur de précipitalion limite pour qu'il y ait écoulement suivant le degré de saturation, c'est-à-dire la pluie d'imbibition, et c'est sur la pluie utile amputée de cette pluie d'imbibition que l'on calcule la capacité d'absorption qui est alors une capacité d'infiltration.

Le résultat de ces opérations conduit à une série de valeurs de la capacité d'absorption ou du coefficient de ruissellement; il s'agit de dé-

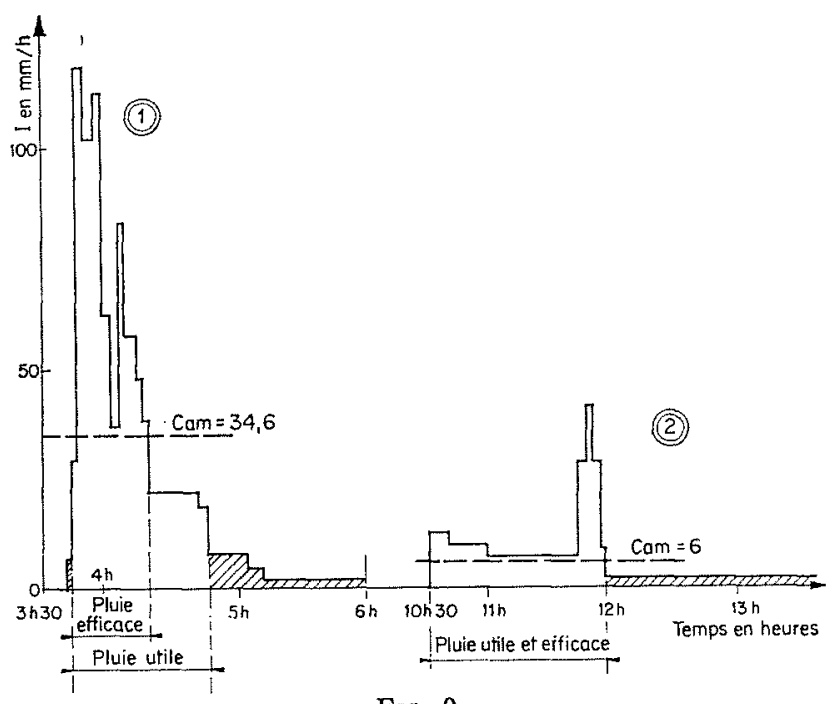

Fig. 9

Variation de la capacité d'absorption. Bassin versant de Koumbaka, averse du 29 juillet 1957 .

Hyétogramme moyen à deux pointes d'averse.

gager les relations entre ces données et les caractéristiques correspondantes des averses génératrices.

Or, ces caractéristiques sont au nombre de cinq ou six, plus ou moins indépendantes et, dans presque tous les cas, l'échantillon dont on dispose est tout à fait insuffisant pour déterminer rigoureusement les relations cherchées. On ne retient que les deux facteurs qui ont la plus forte influence, par exemple l'état de saturation et la hauteur de pluie utile; les autres facteurs donnent lieu à une dispersion notable, comme il est possible de le vérifier sur le graphique 10 .

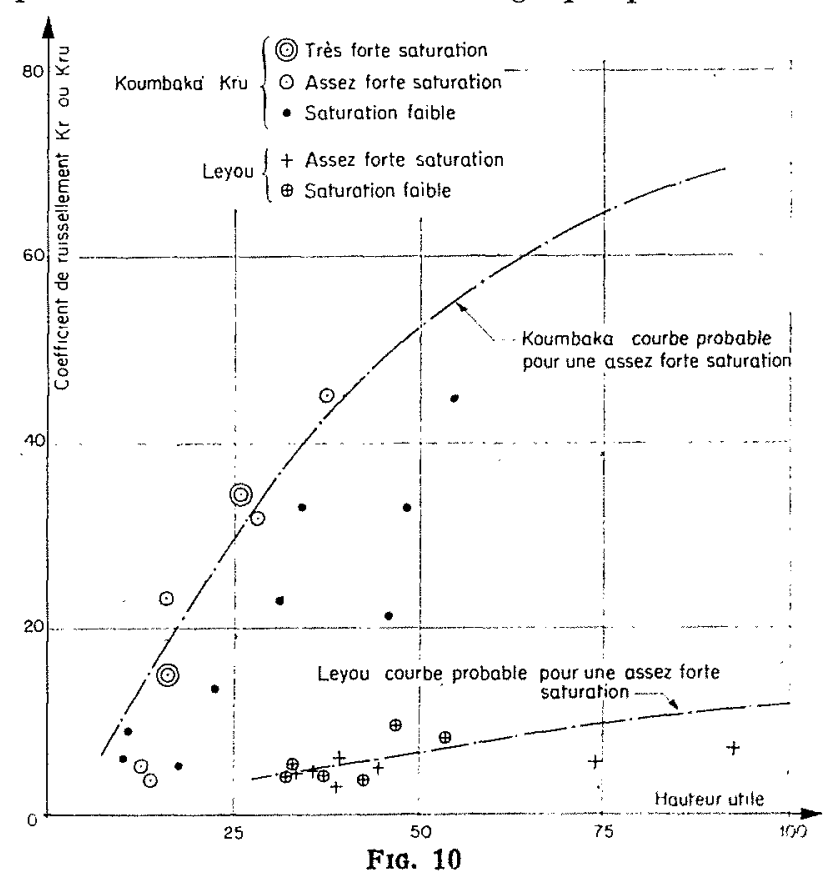

Variation du coefficient de ruissellement en fonction de la hauteur de précipitations. 


\section{Tableav 11}

\begin{tabular}{|c|c|c|c|c|c|c|c|c|c|c|c|c|c|c|c|c|c|c|}
\hline Date & $\begin{array}{c}\text { Crue } \\
\mathrm{N}^{\circ}\end{array}$ & $\begin{array}{l}\text { en } \\
P_{M}\end{array}$ & $\begin{array}{l}\mathrm{mm} \\
\mathrm{P}_{m}\end{array}$ & $P$ & $\begin{array}{c}\mathrm{T}_{u} \\
\mathrm{~m} n \mathrm{n}\end{array}$ & $\begin{array}{r}\mathrm{H}_{u} \\
\mathrm{mnn}\end{array}$ & $\left|\begin{array}{c}\max \\
P \\
n^{\circ} 1\end{array}\right|$ & $\mathrm{VH} \mathrm{m}^{3}$ & $V R m^{3}$ & ${ }_{\%}{ }_{\%}$ & $\begin{array}{c}\% \\
\mathrm{~K}_{r t}\end{array}$ & $\left|\begin{array}{c}Q \\
\max \\
m^{3} / s\end{array}\right|$ & Lag & Rise & $\operatorname{Tr}$ & $\begin{array}{c}\mathrm{CA} \\
\mathrm{mm} / \mathrm{h}\end{array}$ & I & Observations \\
\hline $31-7$ & 25 & 25 & 1 & 11,6 & 17 & 6,9 & 42 & $301.500 \mid$ & 129.500 & 43 & $(72)$ & 14,6 & $\begin{array}{lll}1 & \mathrm{~h} & 30\end{array}$ & $50^{\prime}$ & & $6,7 \mid$ & $2 \mathrm{j}^{\star}$ & Pluie non généralisée \\
\hline $9-8$ & 26 & 44 & $\mid 32$ & $\mid 35,7$ & $|23|$ & $|28,1|$ & $|180|$ & $\mid 928.000$ & 235.000 & $|25,3|$ & $|32,2|$ & $|19,2|$ & |1 h 50 & $11 \mathrm{~h} 33$ & & 47,2 & $2 \mathrm{j}$ & longue traine \\
\hline $14-8 \mid$ & 27 & $\mid 30$ & | 19,9 & $|26,5|$ & $|29|$ & $|22,4|$ & 95 & 689.000 & 75.000 & $\mid 10,9$ & 13 & $|6,6|$ & | 1 h 45 & $1^{x^{\prime}} \mathrm{h} 30$ & & 40,3 & $3 \mathrm{j}$ & \\
\hline $15-8$ & 27 bis & 18,5 & 14 & $|17,3|$ & $|28|$ & 15,2 & 50 & 450.000 & 158.000 & $35,2 \mid$ & 40,1 & $|13,2|$ & $\left|\begin{array}{lll}1 & \mathrm{~h} & 10\end{array}\right|$ & 1 h $\mathrm{h} 20$ & & 19,5 & $\sqrt{12 h^{*}}$ & \\
\hline \multicolumn{19}{|c|}{$\begin{array}{l}1 \mathrm{j} \text { Averse antérieure }<10 \mathrm{~mm} \text {. } \\
1 \mathrm{j} \text { Averse antérieure de } 10 \dot{\mathrm{a}} 20 \mathrm{~mm} \text {. } \\
1 \mathrm{j} \text { Averse antérieure }>20 \mathrm{~mm} . \\
\text { Pluie préliminaire. }\end{array}$} \\
\hline
\end{tabular}

Koumbaka - Station n" II (26 km²) 1957

Mais deux points sont à noter :

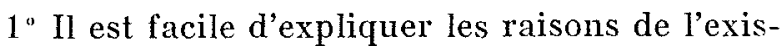
tence de chacun des points aberrants et on peut vérifier effectivement qu'il donne lieu à une valeur trop faible ou trop forte;

$2^{\circ}$ Les nuages de points de deux bassins différents sont netlement distincts : pour une averse de $80 \mathrm{~mm}$ par exemple, le coefficient de ruissellement $K$, est compris entre 9 et $13 \%$ sur le Leyou et $\mathrm{K}_{m}$ varie entre 65 et $75 \%$ sur le bassin de Koumbaka. Pour des bassins de caractéristiques analogues, on aboutit aux mêmes fourchettes.

Dans le cas de terrains relativement perméables, il est possible de guider l'extrapolation des courbes donnant les variations de la capacité d'absorption grâce aux données des essais Muntz ou Porcher.

Enfin, un procédé souvent employé consiste à dresser de grands tableaux des averses les plus importantes, tels que celui du tableau 11 où sont portées les capacités d'absorption ou coefficients de ruissellement et toutes les caractéristiques de l'averse. Par comparaison de ces caractéristiques avec celles de l'averse exceptionnelle, il est possible d'obtenir un ordre de grandeur limite de la capacité d'absorption ou du coefficient de ruissellement. Mais, de toute façon, c'est certainement cette dernière opération qui fait le plus appel au flair de l'hydrologue.

\section{II. - CAlCUL DES DEBitS DE CRUES EXCEPTIONNELLES (Crue décennale)}

Le principe du calcul est le suivant :

On suppose que l'averse décennale présente une hauteur maximale ponctuelle de fréquence décennale et que toutes les autres caracléristiques de cette averse ont les valeurs les plus friquentes pour les fortes averses de cette catégorie.

Toutes les caractéristiques étant déterminées, on applique alors les principes de l'hydrogramme unitaire.

a) Choix des caractéristiques de l'hydrogramme de l'averse :

Il s'agit de déterminer la hauteur maximale ponctuelle, le coefficient de réduction entre le maximum ponctuel et la hauteur moyenne, le hyétogramme et l'état de saturation du sol avant l'averse.

Prenons l'exemple du bassin forestier du Leyou. Il est possible, dans celte région, de remplacer l'étude statistique des averses par celle des précipitations de 24 heures. Calculée sur 400 stations-années, l'averse décennale est de $130 \mathrm{~mm}$.

Le hyétogramme correspondant est déterminé par l'examen des relevés des fortes averses : il comprend un corps à forte intensité, dépassant parfois $120 \mathrm{~mm} / \mathrm{h}$ et une longue traîne à intensité inférieure à $10 \mathrm{~mm} / \mathrm{h}$. La pluie utile est égale à $75 \%$ de la pluie totale.

Pour les régions sahéliennes et subdésertiques, on a établi un catalogue des très fortes averses, 
ce qui facilite le choix de la forme du hyétogramme décennal.

L'examen des coefficients de réduction sur le bassin du Leyou montre qu'il doit être de $90 \%$ pour un maximum ponctuel de $130 \mathrm{~mm}$ (en Mauritanie, M. Brunet-Moret a imaginé un mode de détermination de ce coefficient de réduction que nous ne pouvons pas exposer ici).

Une étude statistique montre que la saturation la plus fréquente, pour de très fortes averses, correspond à un intervalle de trois jours après une première averse de l'ordre de $40 \mathrm{~mm}$.

\section{b) Calcul de la crue exceptionnelle.}

Avec les caractéristiques ci-dessus, la crue décennale se calcule conme suit :

\section{$1^{\circ}$ Bassin du Leyou $\left(6 \mathrm{~km}^{2}\right)$.}

L'averse utile d'une heure est unilaire. La pluie moyenne est de $130 \times 0,90=117 \mathrm{~mm}$.

Le coefficient de ruissellement $K_{1}$. pour une telle averse serait compris entre 8,5 et $12 \%$; par prudence, nous adopterons $11 \%$.

Le volume de ruissellement :

$$
\mathrm{V}_{\mathrm{k}}=0,117 \times 0,11 \times 6 \times 10^{6}=77000 \mathrm{~m}^{3} \text {. }
$$

Le diagramme de distribution donne un débit maximal de $800 \mathrm{l} / \mathrm{s}$ pour $V_{\mathrm{P}}=10000 \mathrm{~m}^{3}$.

Dans ce cas, on peut le remplacer par un triangle.
Le débit maximal calculé est égal à :

$$
0,8 \times \frac{77000}{10000}=6,15 \mathrm{~m}^{3} / \mathrm{s}
$$

En ajoutant un débit hypodermique de $350 \mathrm{l} / \mathrm{s}$, il vient $6,5 \mathrm{~m}^{3} / \mathrm{s}$ pour la crue décennale, soit entre 1000 et $1100 \mathrm{l} / \mathrm{s} . \mathrm{km}^{2}$.

\section{$2^{\circ}$ Bassin de Makabana $\left(2 \mathrm{~km}^{2}\right)$.}

C'est un bassin de savane à écoulement plus rapide. L'averse utile d'une heure n'est plus unitaire. La pluie utile pour une perméabilité plus faible est de $80 \%$ de la pluie totale. Le coefficient de réduction est de $96 \%$. La pluie utile est de $130 \times 0,96 \times 0,80 \mathrm{~mm}$, soit $100 \mathrm{~mm}$; on la divise en deux fractions de 30 minutes, l'une de $40 \mathrm{~mm}$, l'autre de $60 \mathrm{~mm}$.

Le tableau des observations montre que la premiere averse a un coefficient de ruissellement utile de $25 \%$; la seconde, d'après l'examen des pointes multiples, aurait un coefficient de ruissellement de $50 \%$ :

$$
\mathrm{V}_{\mathrm{R} 1}=20000 \mathrm{~m}^{3} \quad \mathrm{~V}_{\mathrm{R} 2}=59000 \mathrm{~m}^{3}
$$

Le premier hydrogramme pour $20000 \mathrm{~m}^{3}$ présente un maximum de $4,3 \mathrm{~m}^{3} / \mathrm{s}$; le second, un maximum de $12,7 \mathrm{~m}^{3} / \mathrm{s}$.

En ajoutant les deux hydrogrammes avec un décalage de 30 minutes, on obtient un volume ruisselé de $14,84 \mathrm{~m}^{3} / \mathrm{s}$.

Il n'y a pas d'écoulement hypodermique sur ce bassin. La crue décennale est donc de l'ordre de $15 \mathrm{~m}^{\mathrm{s}} / \mathrm{s}$, soit $7000-8000 \mathrm{l} / \mathrm{s} \mathrm{km}^{2}$.

\section{CONCLUSION}

La méthode de l'hydrogramme unitaire n'est pas d'un emploi automatique. Tout au long de l'application de la méthode, l'hydrologue doit faire intervenir son jugement, soit pour choisir le procédé le mieux adapté aux conditions du bassin à étudier, soit pour fixer la valeur d'un coefficient à l'intérieur d'une «fourchette » qui est parfois assez large. C'est pourquoi il importe de procéder à de multiples recoupements, de comparer en particulier les résultats définitifs à ceux qui ont été obtenus sur des bassins analogues.

Nous estimons que, par ces méthodes, il est possible d'estimer la crue décennale à 15 ou $20 \%$ près, après trois ans d'observations. Par la simple observation des débits à la station de jaugeage, il faudrait au moins vingt ans de relevés conti- nus pour arriver au même résultat. Encore doilon ajouter que les données obtenues par le second procédé sont loin de fournir, pour les bassins voisins, les mêmes renseignements que peut fournir un bassin équipé pour l'étude de l'hydrogramme unitaire.

L'étude d'un bassin expérimental étant cô̂teuse, il faut la considérer comme un investissement pour la totalité des petits aménagements à prévoir dans un délai de dix à vingt ans, dans une région climatique déterminée.

Bien entendu, il ne faut pas limiter les études sur le terrain à la détermination des crues : l'augmentation des frais sera très faible si on étudie, en complément, les divers éléments du cycle hy. drologique. 


\section{DISCUSSION}

Président : M. DeYmí́

M. le Président observe que M. Rodren applique la méthode de l'hydrogramme unitaire sans tracer d'hydrogramme, en insistant sur le coefficient de ruissellement et sur la capacité d'absorption qui sont les points les plus délicats de la méthode. Il demande à M. Rodier pourquoi, dans un des graphiques projetés, la ligne qui sépare l'écoulement hypodermique des nappes souterraines est ascendante, alors que la courbe de décrue $\varphi=\varphi_{o} e^{-\alpha t}$ est décroissante.

M. Ropren répond que, lorsque l'on étudie le débit de base sur la période des trois mois de saison des pluies, on obtient d'abord une croissance légère. Bien que les courbes de récession présentent une légère pente descendante à une distance suffisante des averses ayant provoqué ruissellement, les niveaux des débits de base vont en croissant d'une averse à l'autre. Vers la fin de la saison des pluies, les débits de base, après chaque averse, sont de plus en plus faibles, de sorte que la courbe générale du débit de base décrô̂t, au contraire. Le phénomène est, en outre, compliqué par des pluies à faible intensité qui interviennent entre deux fortes averses de ruissellement, pluies dont l'influence est difficile à suivre sur les parties très plates de l'hydrosramme. Il peut résulter de l'ensemble du phénomène une courbe de base du diagramme qui soit légèrement ascendante. C'est précisément ce qui s'est produit dans le cas du diagramme projeté, mais ce phénomène est purement accidentel : dans le cas général, le débit de base est, bien entendu, décroissant. M. Rodier reconnait que l'exemple du graphique était mal choisi pour illustrer l'exposé et il indique qu'il sera remplacé par une autre averse plus classique.

M. Bruls demande à M. Ropier s'il juge indispensable, pour le calcul de l'hydrogramme unitaire, de tenil compte de l'écoulement hypodermique. Ne peut-on, comme on le fait aux Etats-Unis, l'englober dans l'écoulement de base?

M. Rodier répond que la méthode utilisée aux EtatsUnis est admissible lorsque le débit hypodermique ne dépasse pas $10 \%$ du débit de base : dans les cas où il est supérieur, l'élimination du débit hypodermique rend fantaisiste l'estimation du temps de base et risque dans certains cas de permettre le choix de valeurs assez arbitraires pour le volume de ruissellement, ce qui est plus grave.

M. le Président remercie M. Rodier.

\section{CALENDRIER DU COMITE TECHNIQUE POUR 1961}

La Société Hydrotechnique de France vous présente ses meilleurs vaux pour l'année 1961 et vous communique le calendrier des activités de son Comité Technique en cette nouvelle année :

- $l^{\text {r" }}$ session : 16 et 17 mars 1961 ,

- $2^{\circ}$ session : 15 et 16 juin 1961,

- $3^{\mathrm{e}}$ session : 16 et 17 novembre $196 \mathrm{~V}$.

Les ordres du jour des sessions de mars, juin et novembre seront communiqués, comme d'habitude, deux semaines avant la date de ces sessions.

Nous pouvons, ici même, indiquer succinctement le programme de la session de mars :

- Jeudi 16 mars (matin) : compte rendu de congrès,

- Jeudi 16 mars (après-midi) : communications diverses;

- Vendredi 17 mars (matin et après-midi) : séances spéciales sur «La mécanique des fluides et la magnétohydrodynamique $\gg$ (élude des modifications des lois hydrodynamiques sous l'effet d'un champ magnétique s'exerçant sur le liquide en mouvement). 